\title{
Intrinsic piezoelectric ferromagnetism with large out-of-plane piezoelectric response in Janus monolayer $\mathrm{CrBr}_{1.5} \mathrm{I}_{1.5}$
}

\author{
San-Dong Guo ${ }^{1}$, Xiao-Shu Guo ${ }^{1}$, Xiu-Xia Cai ${ }^{1}$, Wen-Qi $\mathrm{Mu}^{1}$ and Wen-Cai Ren ${ }^{2,3}$ \\ ${ }^{1}$ School of Electronic Engineering, Xi'an University of Posts and Telecommunications, Xi'an 710121, China \\ ${ }^{2}$ Shenyang National Laboratory for Materials Science, Institute of Metal Research, \\ Chinese Academy of Science, 110016 Shenyang, Liaoning, P. R. China and \\ ${ }^{3}$ School of Materials Science and Engineering, University of Science and Technology of China, Shenyang 110016, P. R. China
}

\begin{abstract}
A two-dimensional (2D) material system with both piezoelectricity and ferromagnetic (FM) order, referred to as a 2D piezoelectric ferromagnetism (PFM), may open up unprecedented opportunities for intriguing physics. Inspired by experimentally synthesized Janus monolayer MoSSe from $\mathrm{MoS}_{2}$, in this work, the Janus monolayer $\mathrm{CrBr}_{1.5} \mathrm{I}_{1.5}$ with dynamic, mechanical and thermal stabilities is predicted, which is constructed from synthesized ferromagnetic $\mathrm{CrI}_{3}$ monolayer by replacing the top I atomic layer with $\mathrm{Br}$ atoms. Calculated results show that monolayer $\mathrm{CrBr}_{1.5} \mathrm{I}_{1.5}$ is an intrinsic FM half semiconductor with valence and conduction bands being fully spin-polarized in the same spin direction. Furthermore, monolayer $\mathrm{CrBr}_{1.5} \mathrm{I}_{1.5}$ possesses a sizable magnetic anisotropy energy (MAE). By symmetry analysis, it is found that both in-plane and out-of-plane piezoelectric polarizations can be induced by a uniaxial strain in the basal plane. The calculated in-plane $d_{22}$ value of $0.557 \mathrm{pm} / \mathrm{V}$ is small. However, more excitingly, the out-of-plane $d_{31}$ is as high as $1.138 \mathrm{pm} / \mathrm{V}$, which is obviously higher compared with ones of other 2D known materials. The strong out of-plane piezoelectricity is highly desirable for ultrathin piezoelectric devices. Moreover, strain engineering is used to tune piezoelectricity of monolayer $\mathrm{CrBr}_{1.5} \mathrm{I}_{1.5}$. It is found that compressive strain can improve the $d_{22}$, and tensile strain can enhance the $d_{31}$. A FM order to antiferromagnetic (AFM) order phase transition can be induced by compressive strain, and the critical point is about 0.95 strain. That is to say that a 2D piezoelectric antiferromagnetism (PAFM) can be achieved by compressive strain, and the corresponding $d_{22}$ and $d_{31}$ are $0.677 \mathrm{pm} / \mathrm{V}$ and $0.999 \mathrm{pm} / \mathrm{V}$ at 0.94 strain, respectively. It is also found that magnetic order has important effects on piezoelectricity of monolayer $\mathrm{CrBr}_{1.5} \mathrm{I}_{1.5}$. Finally, similar to $\mathrm{CrBr}_{1.5} \mathrm{I}_{1.5}$, the $\mathrm{PFM}$ can also be realized in the monolayer $\mathrm{CrF}_{1.5} \mathrm{I}_{1.5}$ and $\mathrm{CrCl}_{1.5} \mathrm{I}_{1.5}$. Amazingly, their $d_{31}$ can reach up to $2.578 \mathrm{pm} / \mathrm{V}$ and $1.804 \mathrm{pm} / \mathrm{V}$ for monolayer $\mathrm{CrF}_{1.5} \mathrm{I}_{1.5}$ and $\mathrm{CrCl}_{1.5} \mathrm{I}_{1.5}$. Our works propose a realistic way to achieve PFM with large $d_{31}$, making these systems very promising for multifunctional semiconductor spintronic applications.
\end{abstract}

PACS numbers: 71.20.-b, 77.65.-j, 72.15.Jf, 78.67.-n

Email:sandongyuwang@163.com Keywords: Ferromagnetism, Piezoelectronics, 2D materials

\section{INTRODUCTION}

The piezoelectric effect is an intrinsic electromechanical coupling in semiconductors with crystal structures lacking inversion symmetry. The reduction in dimensionality of $2 \mathrm{D}$ materials often can eliminate inversion symmetry, which allows them to be piezoelectric. Experimentally, the piezoelectricity of $\mathrm{MoS}_{2}{ }^{1,2}, \mathrm{MoSSe}^{3}$ and $\mathrm{In}_{2} \mathrm{Se}_{3}{ }^{4}$ monolayers have been discovered, which pushes the development of piezoelectric properties of $2 \mathrm{D}$ materials. In theory, many kinds of $2 \mathrm{D}$ materials have been predicted to be piezoelectric by density functional theory (DFT) calculations ${ }^{5-12}$. The strain-tuned piezoelectric response has also been investigated by DFT calculations, and it is proved that strain can improve the piezoelectric strain coefficients ${ }^{13-15}$.

Great advances have been made on 2D piezoelectric materials. However, there are two main issues of 2D piezoelectric materials. One is that most $2 \mathrm{D}$ materials possess solely piezoelectricity. The multifunctional 2D materials, such as combination of piezoelectricity with topological insulating phase or ferromagnetism, are of particular interest, whose exploitation may promise novel device applications. The coexistence of intrinsic piezo- electricity and ferromagnetism has been predicted in $2 \mathrm{D}$ vanadium dichalcogenides and $\mathrm{VSi}_{2} \mathrm{P}_{4}{ }^{16,17}$. The piezoelectric quantum spin Hall insulators (PQSHI) have also been achieved in monolayer InXO $(\mathrm{X}=\mathrm{Se} \text { and } \mathrm{Te})^{18}$ and Janus monolayer $\mathrm{SrAlGaSe}_{4}{ }^{19}$. Another is that the outof-plane piezoelectricity in known 2D materials is absent or weak. The strong out-of-plane piezoelectric effect and its inverse effect are highly desirable for piezoelectric devices, which is compatible with the bottom/top gate technologies. Many strategies have been made for searching 2D piezoelectric materials with large $d_{31}$ or $d_{32} 5,11,20-22$. A significant improvement is that the piezoelectric strain coefficient $d_{31}$ of $\mathrm{Sc}_{2} \mathrm{CO}_{2}$ MXene is up to $0.78 \mathrm{pm} / \mathrm{V}^{22}$.

A natural idea is to search for multifunctional 2D piezoelectric materials with large out-of-plane piezoelectricity. A few types of $2 \mathrm{D}$ magnetic materials have been studied ${ }^{23-31}$. For example, the monolayer $\mathrm{Cr}_{2} \mathrm{Ge}_{2} \mathrm{Te}_{6}$, $\mathrm{VS}_{2}$ and $\mathrm{VSe}_{2}$ have been experimentally proved to magnetic materials ${ }^{25,28}$. The Dirac spin-gapless semiconductor (SGS) with $100 \%$ spin polarization, high Fermi velocities and high Curie temperatures has been predicted in $\mathrm{Mn}_{2} \mathrm{C}_{6} \mathrm{Se}_{12}$ and $\mathrm{Mn}_{2} \mathrm{C}_{6} \mathrm{~S}_{6} \mathrm{Se}_{6}$ monolayers ${ }^{29}$. The $2 \mathrm{D}$ high-temperature ferromagnetic half-metal (FMHM) can be realized in transition-metal embedded carbon nitride 


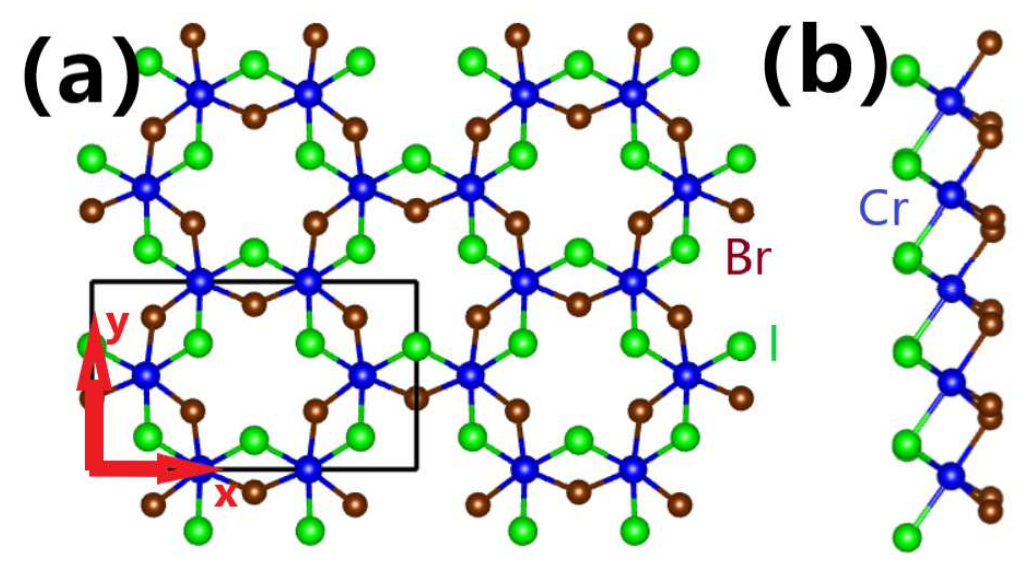

FIG. 1. (Color online)The (a) top view and (b) side view of crystal structure of Janus monolayer $\mathrm{CrBr}_{1.5} \mathrm{I}_{1.5}$. The rectangle supercell is marked by black frame, which is used to calculate the piezoelectric stress coefficients. The rectangle's width and height are defined as $\mathrm{x}$ and $\mathrm{y}$ directions, respectively.
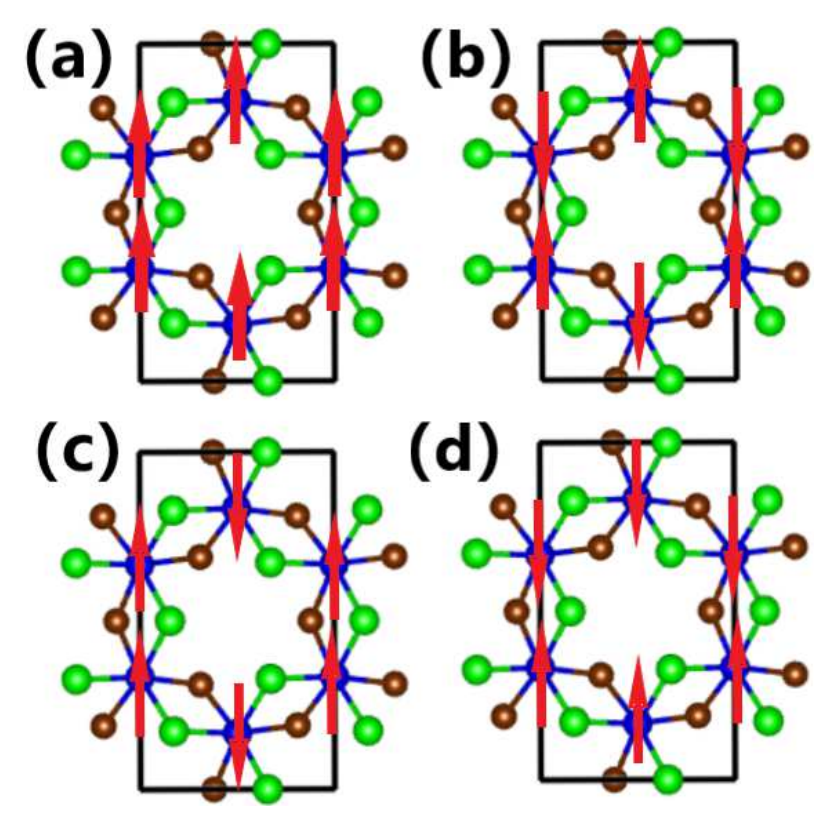

FIG. 2. (Color online)The four considered magnetic configuration of Janus monolayer $\mathrm{CrBr}_{1.5} \mathrm{I}_{1.5}$ : FM (a), AF-Néel (b), AF-zigzag (c), and AF-stripy ordered (d). The crystal cells used in the calculations are marked with red arrows as the spin direction of $\mathrm{Cr}$ atoms.

monolayers ${ }^{27}$. The $\mathrm{CrI}_{3}$ monolayer is firstly predicted to be FM order by the first-principle calculations ${ }^{32}$, and then is confirmed experimentally ${ }^{30}$. A series of studies have been carried out to explore the magnetic related properties in $\mathrm{CrI}_{3}$ monolayer ${ }^{33-38}$. These provide many new possibilities to combine the piezoelectricity and magnetism into the same kind of 2D material.

It is noted that the $\mathrm{CrI}_{3}$ monolayer has sandwiched I-Cr-I structure with inversion symmetry, and then possesses no piezoelectricity. However, it is possible to construct Janus structure based on $\mathrm{CrI}_{3}$ monolayer, and then produce piezoelectric effect. Janus monolayer MoSSe has

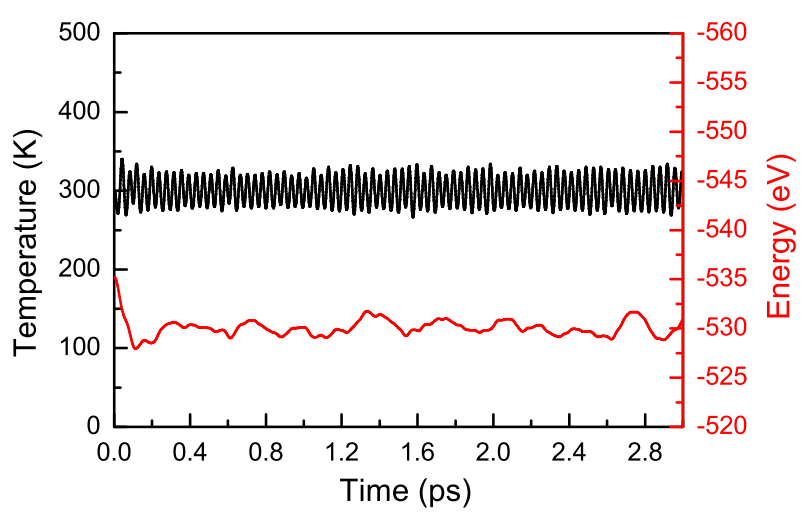

FIG. 3. (Color online) The temperature and total energy fluctuations of Janus monolayer $\mathrm{CrBr}_{1.5} \mathrm{I}_{1.5}$ with FM magnetic configuration at $300 \mathrm{~K}$.

been synthesized experimentally from $\mathrm{MoS}_{2}$ monolayer by breaking the out-of-plane structural symmetry ${ }^{39}$. That is, the Janus monolayer MoSSe can be constructed by replacing one of two $\mathrm{S}$ layers with Se atoms in $\mathrm{MoS}_{2}$ monolayer. In this work, Janus monolayer $\mathrm{CrBr}_{1.5} \mathrm{I}_{1.5}$ is constructed from synthesized ferromagnetic $\mathrm{CrI}_{3}$ monolayer by replacing the top I atomic layer with $\mathrm{Br}$ atoms, which is dynamically, mechanically and thermally stable. It is found that monolayer $\mathrm{CrBr}_{1.5} \mathrm{I}_{1.5}$ is an intrinsic FM half semiconductor with a sizable MAE. Although the calculated in-plane $d_{22}(0.557 \mathrm{pm} / \mathrm{V})$ is small, the outof-plane $d_{31}(1.138 \mathrm{pm} / \mathrm{V})$ is very large, which is obviously higher than ones of other $2 \mathrm{D}$ known materials. It is proved that strain engineering can effectively tune piezoelectricity of monolayer $\mathrm{CrBr}_{1.5} \mathrm{I}_{1.5}$. A 2D PAFM can also be achieved in monolayer $\mathrm{CrBr}_{1.5} \mathrm{I}_{1.5}$ by compressive strain, and the calculated results show that magnetic order has important influences on piezoelectricity of monolayer $\mathrm{CrBr}_{1.5} \mathrm{I}_{1.5}$. It is also proved that the PFM can also be achieved in monolayer $\mathrm{CrF}_{1.5} \mathrm{I}_{1.5}$ and monolayer $\mathrm{CrCl}_{1.5} \mathrm{I}_{1.5}$, which show very large $d_{31}$ of $2.578 \mathrm{pm} / \mathrm{V}$ and 

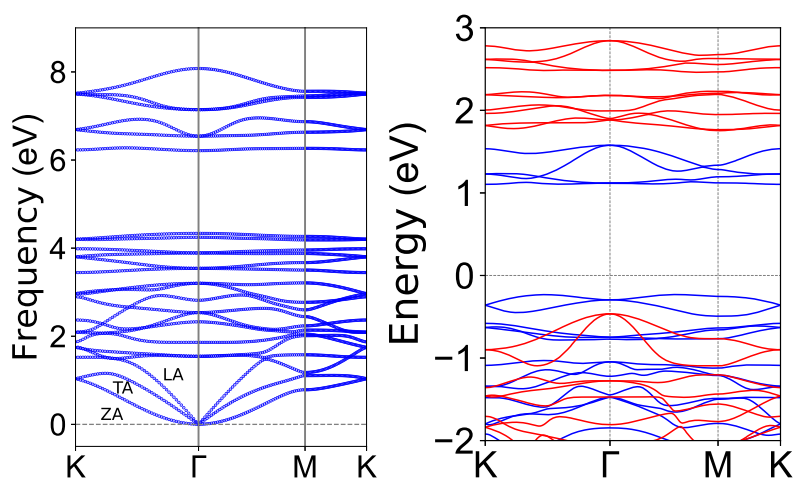

FIG. 4. (Color online)Left: the phonon band dispersions of Janus monolayer $\mathrm{CrBr}_{1.5} \mathrm{I}_{1.5}$ with FM magnetic configuration. Right: the energy band structures of $\mathrm{CrBr}_{1.5} \mathrm{I}_{1.5}$ with FM state. The blue (red) lines represent the band structure in the spin-up (spin-down) direction.

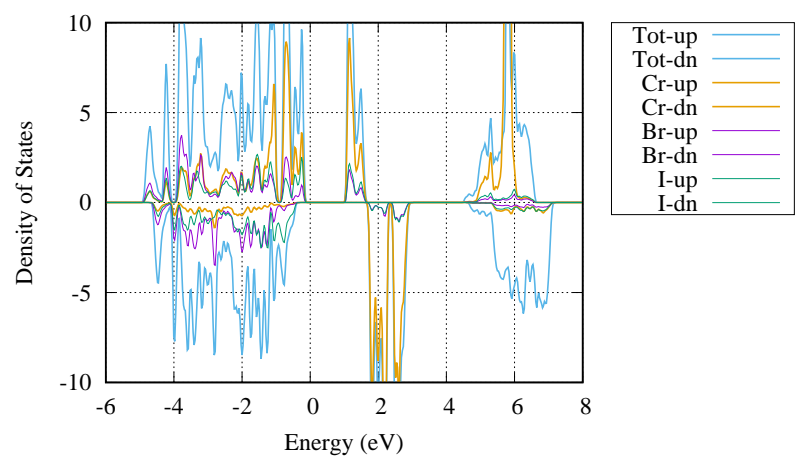

FIG. 5. (Color online) Calculated total and atomic partial density of states of Janus monolayer $\mathrm{CrBr}_{1.5} \mathrm{I}_{1.5}$ with $\mathrm{FM}$ magnetic configuration.

\section{$1.804 \mathrm{pm} / \mathrm{V}$.}

The rest of the paper is organized as follows. In the next section, we shall give our computational details and methods. In the next few sections, we shall present structural stabilities, electronic structures, and piezoelectric properties of monolayer $\mathrm{CrBr}_{1.5} \mathrm{I}_{1.5}$, along with strain effects on its piezoelectric properties. Finally, we shall give our discussion and conclusions.

\section{COMPUTATIONAL DETAIL}

Within $\mathrm{DFT}^{40}$, we perform the main calculations with spin-polarization using the the plane-wave code VASP $^{41-43}$ within the projector augmented-wave (PAW) method. The popular generalized gradient approximation of Perdew, Burke and Ernzerhof (GGA-PBE) ${ }^{44}$ is used as the exchange-correlation functional. The kinetic energy cutoff is set to $500 \mathrm{eV}$ with the total energy convergence criterion for $10^{-8} \mathrm{eV}$. All the lattice constants and atomic coordinates are optimized until the force on each atom is less than $0.0001 \mathrm{eV} . \AA^{-1}$. A vacuum spacing

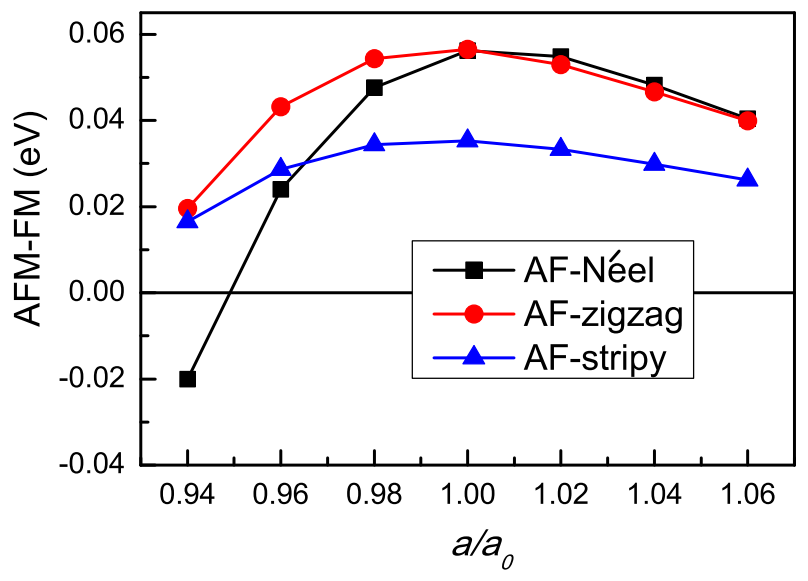

FIG. 6. (Color online) Calculated energy differences of AFNéel, AF-zigzag and AF-stripy with respect to FM state as a function of strain with rectangle supercell.

of more than $18 \AA$ is used to avoid interactions between two neighboring images. The elastic stiffness tensor $C_{i j}$ and piezoelectric stress tensor $e_{i j}$ are carried out by using strain-stress relationship (SSR) and density functional perturbation theory (DFPT) method $^{45}$, respectively. A Monkhorst-Pack k-mesh of $8 \times 8 \times 1$ is used to sample the Brillouin Zone (BZ) for the calculations of electronic structure and elastic coefficients $C_{i j}$, and a mesh of $4 \times 8 \times 1 \mathrm{k}$-points for the energy of different magnetic configurations and piezoelectric stress coefficients $e_{i j}$. The $2 \mathrm{D}$ elastic coefficients $C_{i j}^{2 D}$ and piezoelectric stress coefficients $e_{i j}^{2 D}$ have been renormalized by $C_{i j}^{2 D}=L z C_{i j}^{3 D}$ and $e_{i j}^{2 D}=L z e_{i j}^{3 D}$, where the $L z$ is the length of unit cell along $\mathrm{z}$ direction. By finite displacement method, the interatomic force constants (IFCs) are obtained based on the $4 \times 4 \times 1$ supercell with FM ground state. Based on the harmonic IFCs, the phonon dispersions are evaluated using Phonopy code ${ }^{46}$.

\section{STRUCTURE AND STABILITY}

The structure of Janus monolayer $\mathrm{CrBr}_{1.5} \mathrm{I}_{1.5}$ is similar to monolayer $\mathrm{CrI}_{3}$ monolayer, which contains three atomic sublayers with $\mathrm{Cr}$ layer sandwiched between $\mathrm{Br}$ and I layers. It is well known that Janus transition metal dichalchogenides (TMD) Monolayer MoSSe has been synthesized by replacing the top $\mathrm{S}$ atomic layer in $\mathrm{MoS}_{2}$ with Se atoms ${ }^{39}$. Using the same idea, the Janus monolayer $\mathrm{CrBr}_{1.5} \mathrm{I}_{1.5}$ can be constructed by replacing one of two I layers with $\mathrm{Br}$ atoms in monolayer $\mathrm{CrI}_{3}$. The schematic crystal structures of Janus monolayer $\mathrm{CrBr}_{1.5} \mathrm{I}_{1.5}$ are shown in Figure 1. The monolayer $\mathrm{CrI}_{3}$ has centrosymmetry with $\overline{3} m$ point-group symmetry (No.162), but monolayer $\mathrm{CrBr}_{1.5} \mathrm{I}_{1.5}$ loses centrosymmetry and horizontal mirror symmetry with $3 \mathrm{~m}$ point-group symmetry (No.157), which will induce both in-plane and out-of-plane piezoelectricity. 

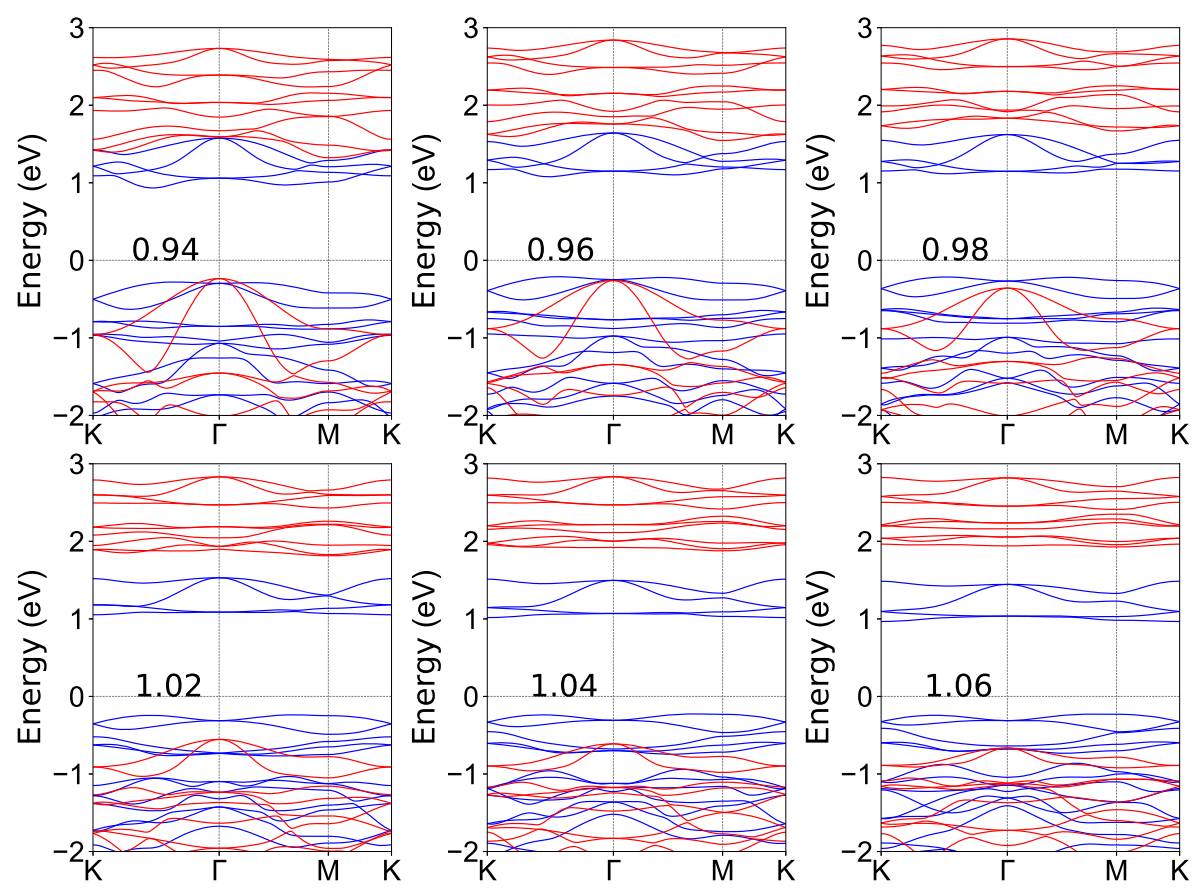

FIG. 7. (Color online) The energy band structures of Janus monolayer $\mathrm{CrBr}_{1.5} \mathrm{I}_{1.5}$ with FM magnetic configuration with $a / a_{0}$ changing from 0.94 to 1.06 .

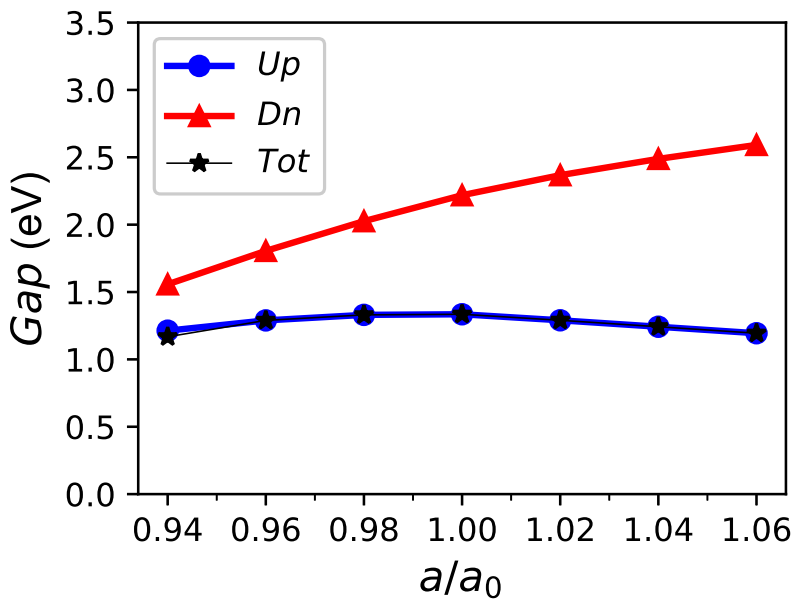

FIG. 8. (Color online) The majority-spin gap $(U p)$, the minority-spin gap $(D n)$ and the total gap (Tot) of FM $\mathrm{CrBr}_{1.5} \mathrm{I}_{1.5}$ as a function of strain $a / a_{0}$.

Four different magnetic configurations (Figure 2) are considered to evaluate the magnetic ground state of monolayer $\mathrm{CrBr}_{1.5} \mathrm{I}_{1.5}$, which are used to investigate the ground state of monolayer $\mathrm{CrX}_{3}(\mathrm{X}=\mathrm{F}, \mathrm{Cl}, \mathrm{Br} \text { and } \mathrm{I})^{32}$. The energy of AF-Néel, AF-zigzag, AF-stripy state and non-magnetic (NM) state with respect to FM state are $56.1 \mathrm{meV}, 56.4 \mathrm{meV}, 35.2 \mathrm{meV}$ and $6.802 \mathrm{eV}$ with rectangle supercell. Our calculated results show that the FM order is the most stable magnetic state. This means that ferromagnetism in monolayer $\mathrm{CrI}_{3}$ is retained by elements substitution to construct Janus structure. The optimized lattice constants with FM state is $6.744 \AA$, which falls between those of the $\mathrm{CrBr}_{3}(6.433 \AA)$ and $\mathrm{CrI}_{3}(7.008 \AA)$ monolayers ${ }^{32}$. For monolayer $\mathrm{CrBr}_{1.5} \mathrm{I}_{1.5}$, the difference in atomic sizes and electronegativities of $\mathrm{Br}$ and I atoms leads to inequivalent $\mathrm{Cr}-\mathrm{Br}$ and $\mathrm{Cr}-\mathrm{I}$ bond lengths (Br$\mathrm{Cr}-\mathrm{Br}$ and I-Cr-I bond angles), and they are $2.542 \AA$ and $2.719 \AA$ (92.036 and 89.432), which can induce a built-in electric field.

The ab initio molecular dynamics (AIMD) simulations using NVT ensemble are performed to assess the thermal stability of the monolayer $\mathrm{CrBr}_{1.5} \mathrm{I}_{1.5}$ at room temperature. Figure 3 shows the temperature and total energy fluctuations of $\mathrm{CrBr}_{1.5} \mathrm{I}_{1.5}$ monolayer as a function of the simulation time. Calculated results show no obvious structural disruption with the temperature and total energy fluctuates being small at the end of the MD simulation at $300 \mathrm{~K}$, which confirms the thermodynamical stability of the $\mathrm{CrBr}_{1.5} \mathrm{I}_{1.5}$ monolayer at room temperature.

The dynamical stability of the $\mathrm{CrBr}_{1.5} \mathrm{I}_{1.5}$ monolayer is analyzed by the phonon spectra, which is plotted in Figure 4 . There are twenty-one optical and three acoustical phonon branches with a total of twenty-four branches due to eight atoms per cell. The longitudinal acoustic (LA) and transverse acoustic (TA) modes mean in-plane vibrations, while the ZA branch represents the out-ofplane vibrations. It is clearly seen that the $\mathrm{ZA}$ branch is quadratic near the zone center, as typical characteristics of $2 \mathrm{D}$ materials ${ }^{47,48}$. All phonon frequencies are positive, confirming the dynamical stability of $\mathrm{CrBr}_{1.5} \mathrm{I}_{1.5}$ monolayer, which means that it can exist as a free-standing 

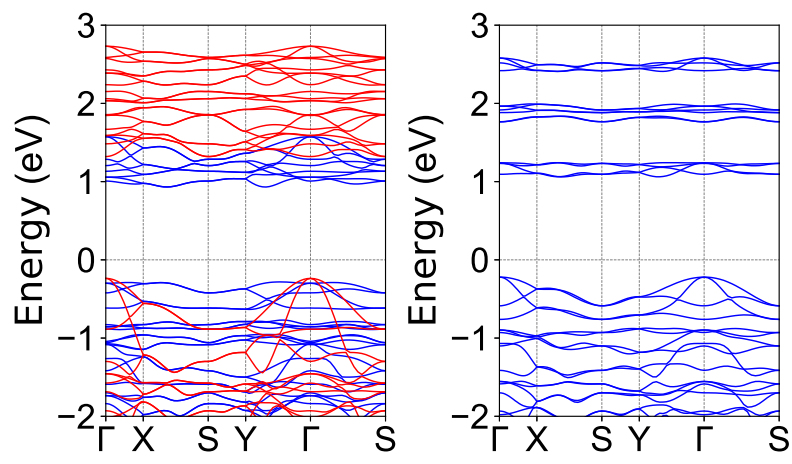

FIG. 9. (Color online)The energy band structures of $\mathrm{CrBr}_{1.5} \mathrm{I}_{1.5}$ with FM (Left) and AF-Néel (Right) states at 0.94 strain. The blue (red) lines represent the band structure in the spin-up (spin-down) direction.

\section{$2 \mathrm{D}$ crystal.}

It is important to check the mechanical stability of $\mathrm{CrBr}_{1.5} \mathrm{I}_{1.5}$ monolayer for practical application. Therefore, we calculate elastic constants using the SSR method. Using Voigt notation, the elastic tensor with $\overline{3} m$ point-group symmetry for $2 \mathrm{D}$ materials can be expressed as:

$$
C=\left(\begin{array}{ccc}
C_{11} & C_{12} & 0 \\
C_{12} & C_{11} & 0 \\
0 & 0 & \left(C_{11}-C_{12}\right) / 2
\end{array}\right)
$$

The calculated $C_{11}$ and $C_{12}$ are $29.75 \mathrm{Nm}^{-1}$ and 8.26 $\mathrm{Nm}^{-1}$, which are between ones of $\mathrm{CrBr}_{3}$ and $\mathrm{CrI}_{3}$ monolayers $^{32}$. The calculated $C_{11}>0$ and $C_{11}-C_{12}>0$ satisfy the Born criteria of mechanical stability ${ }^{49}$, confirming the mechanical stability of $\mathrm{CrBr}_{1.5} \mathrm{I}_{1.5}$ monolayer. We also calculate the Young's moduli $C_{2 D}$, shear modulus $G_{2 D}$ and Poisson's ratio $\nu$ using the method suggested by Andrew et al, and they are $27.46 \mathrm{Nm}^{-1}, 10.75 \mathrm{Nm}^{-1}$ and 0.278 , respectively. These indicate that monolayer $\mathrm{CrBr}_{1.5} \mathrm{I}_{1.5}$ can be easily tuned by strain, which is favorable for novel flexible piezotronics and nanoelectronics.

\section{ELECTRONIC STRUCTURE}

To exhibit piezoelectricity, the monolayer $\mathrm{CrBr}_{1.5} \mathrm{I}_{1.5}$ not only should lack inversion symmetry, but also should be a semiconductor. So, we investigate the electronic structures of $\mathrm{CrBr}_{1.5} \mathrm{I}_{1.5}$ monolayer with $\mathrm{FM}$ ground state, and the energy bands and atomic partial density of states (DOS) are plotted in Figure 4 and Figure 5, respectively. It is found that $\mathrm{CrBr}_{1.5} \mathrm{I}_{1.5}$ monolayer is an indirect gap semiconductor with gap value of $1.335 \mathrm{eV}$. Moreover, the valence and conduction bands near the Fermi level are exclusively contributed by the same spin-up component, showing a typical half-semiconductor character. The difference of the band edge energy between the two spin components for the conduction band minimum $(\mathrm{CBM})$ and the valance band maximum $(\mathrm{VBM})$ are
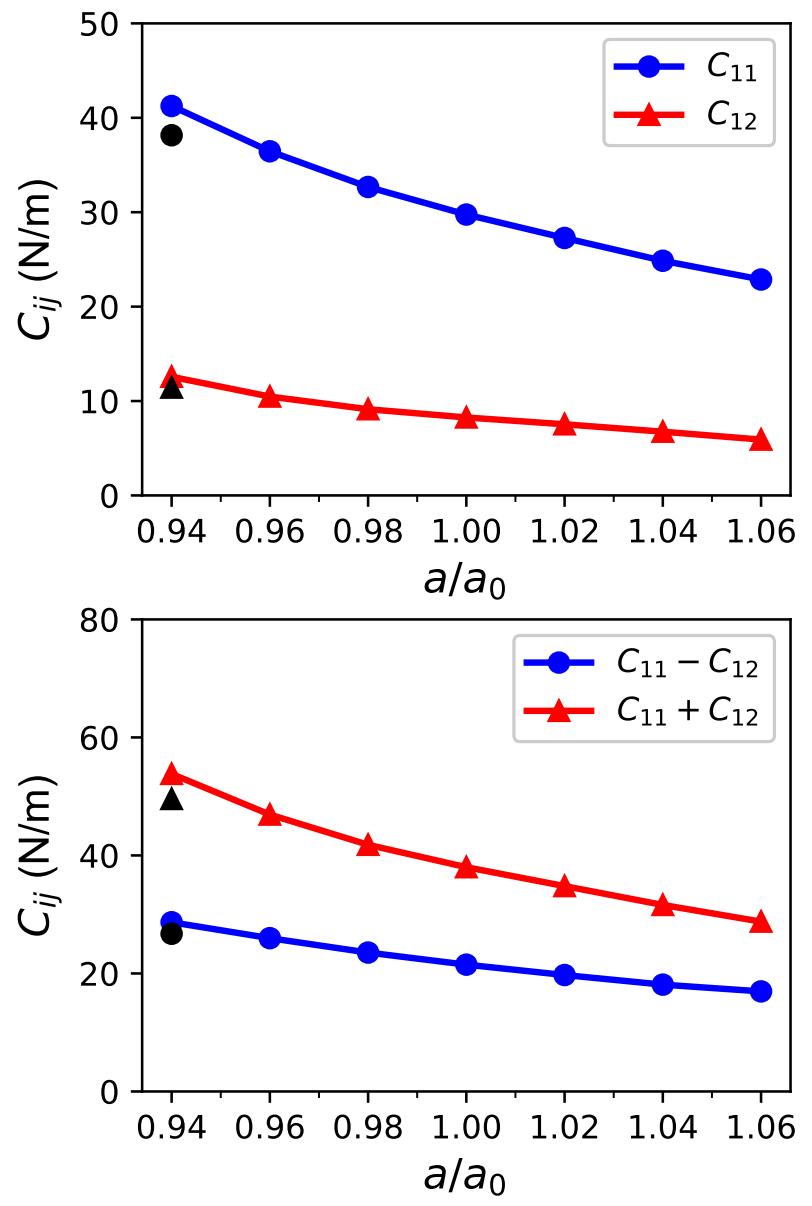

FIG. 10. (Color online) For Janus monolayer $\mathrm{CrBr}_{1.5} \mathrm{I}_{1.5}$ with FM state, the elastic constants $C_{i j}$ with the application of biaxial strain (0.94 to 1.06). The black marks mean AF-Néel results at 0.94 strain.

$0.884 \mathrm{eV}$ and $0.233 \mathrm{eV}$, respectively. According to DOS, the occupied $\mathrm{Cr}-3 d$ orbitals are mainly found in the spinup direction, and the spin-down Cr-3d states are almost unoccupied. For both spin directions, the conduction band is dominated by Cr-3d states, which are weakly hybridized with the $\mathrm{Br}-3 p$ and I- $3 p$ states. In the spin-down direction, the valence band are almost pure $\mathrm{Br}-3 p$ and I$3 p$ character. For the spin-up direction of the valence band, the states are contributed by the $\mathrm{Br}-3 p$ and I- $3 p$ states with a mixture of Cr-3d states. In fact, many electronic properties of monolayer $\mathrm{CrBr}_{1.5} \mathrm{I}_{1.5}$ are similar to ones of $\mathrm{CrI}_{3}$ monolayer ${ }^{32}$.

The magnetic moment of primitive cell is equal to 6 $\mu_{B}$ accurately, which is consistent with its semiconducting property. The local magnetic moments of $\mathrm{Cr}$ is 2.985 $\mu_{B}$, which suggests that monolayer $\mathrm{CrBr}_{1.5} \mathrm{I}_{1.5}$ is robust intrinsic ferromagnetic $2 \mathrm{D}$ semiconductor with large magnetic moments. MAE is an important parameter to confirm ferromagnetic behavior of monolayer $\mathrm{CrBr}_{1.5} \mathrm{I}_{1.5}$. The small MAE will result in superparamagnetic rather than ferromagnetic behavior. By using GGA+spin orbital coupling (SOC), it is found that an easy axis is 

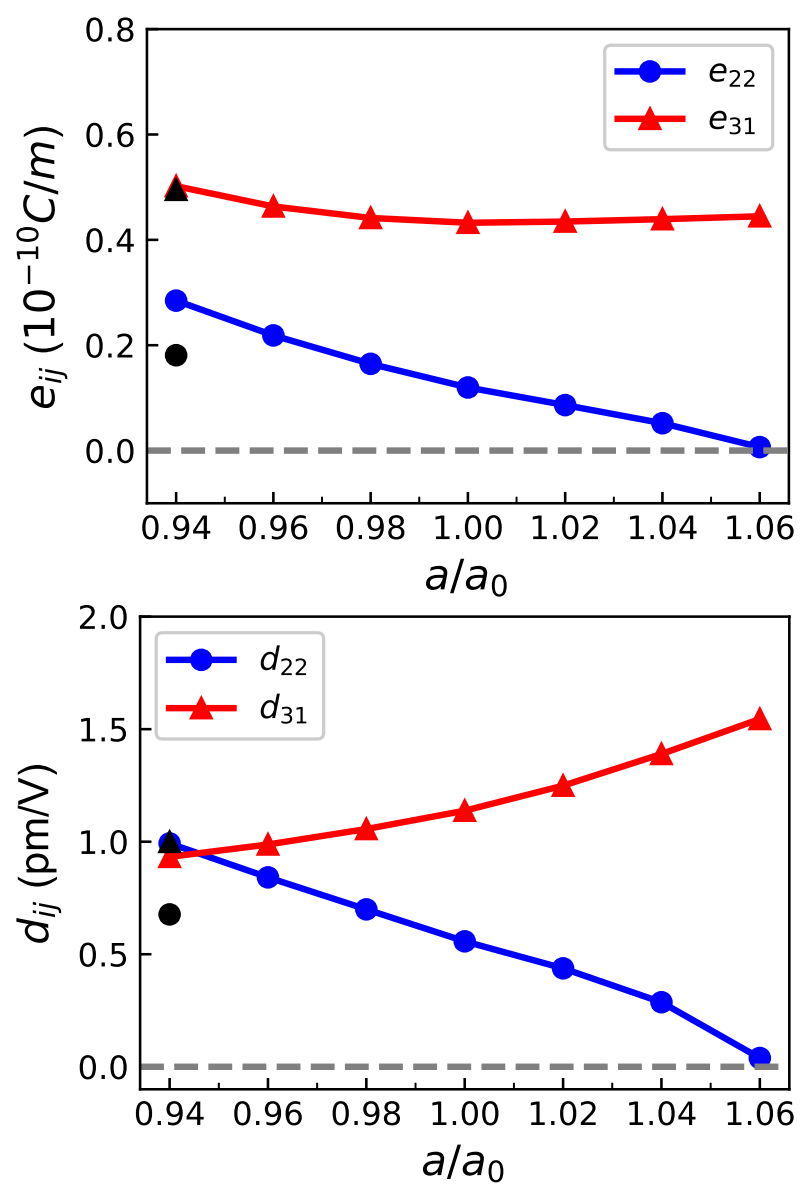

FIG. 11. (Color online) For Janus monolayer $\mathrm{CrBr}_{1.5} \mathrm{I}_{1.5}$ with FM state, the piezoelectric stress coefficients $\left(e_{22}\right.$ and $\left.e_{31}\right)$ and the piezoelectric strain coefficients $\left(d_{22}\right.$ and $\left.d_{31}\right)$ with the application of biaxial strain (0.94 to 1.06). The black marks mean AF-Néel results at 0.94 strain.

along the c-direction for monolayer $\mathrm{CrBr}_{1.5} \mathrm{I}_{1.5}$, and the corresponding MAE is $356 \mu \mathrm{eV}$ per Cr atom. For $\mathrm{CrBr}_{3}$ and $\mathrm{CrI}_{3}$ monolayers, the easy axis is also along the cdirection, and the MAE of monolayer $\mathrm{CrBr}_{1.5} \mathrm{I}_{1.5}$ is between ones of them $\left(185.5 \mu \mathrm{eV}\right.$ per $\mathrm{Cr}$ atom for $\mathrm{CrBr}_{3}$ and $685.5 \mu \mathrm{eV}$ per $\mathrm{Cr}$ atom for $\left.\mathrm{CrI}_{3}\right)^{32}$.

\section{PIEZOELECTRIC PROPERTIES}

The $\mathrm{CrI}_{3}$ monolayer with $\overline{3} m$ point-group symmetry are centrosymmetric, showing no piezoelectricity. The $\mathrm{CrBr}_{1.5} \mathrm{I}_{1.5}$ monolayer with $3 \mathrm{~m}$ point-group symmetry lacks both inversion symmetry and reflectional symmetry across the xy plane, which means that both $e_{22} / d_{22}$ and $e_{31} / d_{31}$ with defined $\mathrm{x}$ and $\mathrm{y}$ direction in Figure 1 are nonzero. For $2 \mathrm{D}$ materials, only the in-plane strain and stress are taken into account ${ }^{5-12}$, and the piezoelectric stress and strain tensors by using Voigt notation can
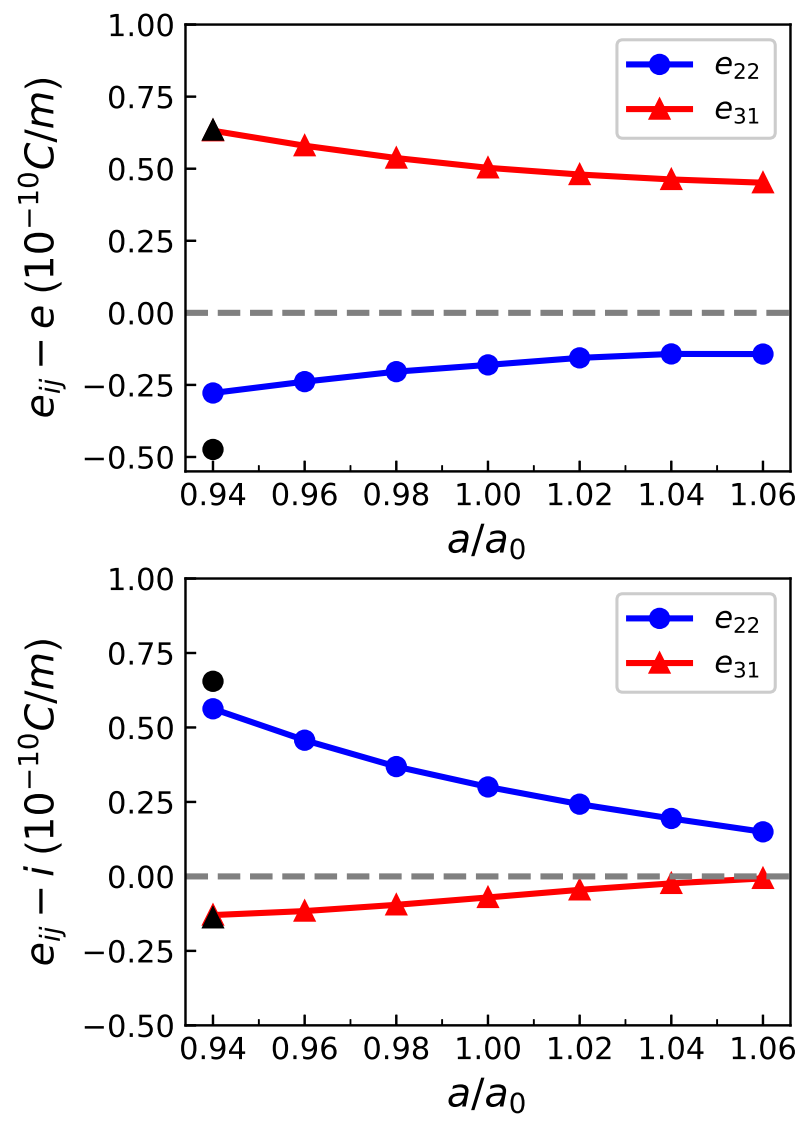

FIG. 12. (Color online) For Janus monolayer $\mathrm{CrBr}_{1.5} \mathrm{I}_{1.5}$ with FM state, the ionic contribution and electronic contribution to $e_{22}$ and $e_{31}$ with the application of biaxial strain (0.94 to 1.06). The black marks mean AF-Néel results at 0.94 strain.

become:

$$
\begin{aligned}
& e=\left(\begin{array}{ccc}
0 & 0 & -e_{22} \\
-e_{22} & e_{22} & 0 \\
e_{31} & e_{31} & 0
\end{array}\right) \\
& d=\left(\begin{array}{ccc}
0 & 0 & -2 d_{22} \\
-d_{22} & d_{22} & 0 \\
d_{31} & d_{31} & 0
\end{array}\right)
\end{aligned}
$$

With a applied uniaxial in-plane strain, both in-plane and vertical piezoelectric polarization $\left(e_{22} / d_{22} \neq 0\right.$ and $\left.e_{31} / d_{31} \neq 0\right)$ can be produced. However, by imposing biaxial in-plane strain, the out-of-plane one still will remain, while the in-plane piezoelectric response will be $\operatorname{suppressed}\left(e_{11} / d_{11}=0\right.$ and $\left.e_{31} / d_{31} \neq 0\right)$. The independent $d_{22}$ and $d_{31}$ are can be attained by $e_{i k}=d_{i j} C_{j k}$ :

$$
d_{22}=\frac{e_{22}}{C_{11}-C_{12}} \quad \text { and } \quad d_{31}=\frac{e_{31}}{C_{11}+C_{12}}
$$

The orthorhombic supercell is used as the computational unit cell (in Figure 1) to calculate the 
TABLE I. For $\mathrm{CrX}_{1.5} \mathrm{I}_{1.5}(\mathrm{X}=\mathrm{F}, \mathrm{Cl}$ and $\mathrm{Br})$ monolayers, the lattice constants $a_{0}(\AA)$, the elastic constants $C_{i j}$ in $\mathrm{Nm}^{-1}$, the piezoelectric stress coefficients $e_{i j}$ in $10^{-10} \mathrm{C} / \mathrm{m}$, the piezoelectric strain coefficients $d_{i j}$ in pm/V, MAE in $\mu e V / \mathrm{Cr}$ and easy axis (EA).

\begin{tabular}{cccccccccc}
\hline \hline Name & $a_{0}$ & $C_{11}$ & $C_{12}$ & $e_{22}$ & $e_{31}$ & $d_{22}$ & $d_{31}$ & MAE & EA \\
\hline $\mathrm{CrF}_{1.5} \mathrm{I}_{1.5}$ & 6.250 & 49.97 & 16.34 & 1.339 & 1.710 & 3.983 & 2.578 & 2151 & $a b$ \\
\hline $\mathrm{CrCl}_{1.5} \mathrm{I}_{1.5}$ & 6.590 & 34.88 & 9.94 & 0.238 & 0.809 & 0.956 & 1.804 & 110 & $c$ \\
\hline $\mathrm{CrBr}_{1.5} \mathrm{I}_{1.5}$ & 6.744 & 29.75 & 8.26 & 0.119 & 0.432 & 0.557 & 1.138 & 356 & $c$ \\
\hline \hline
\end{tabular}
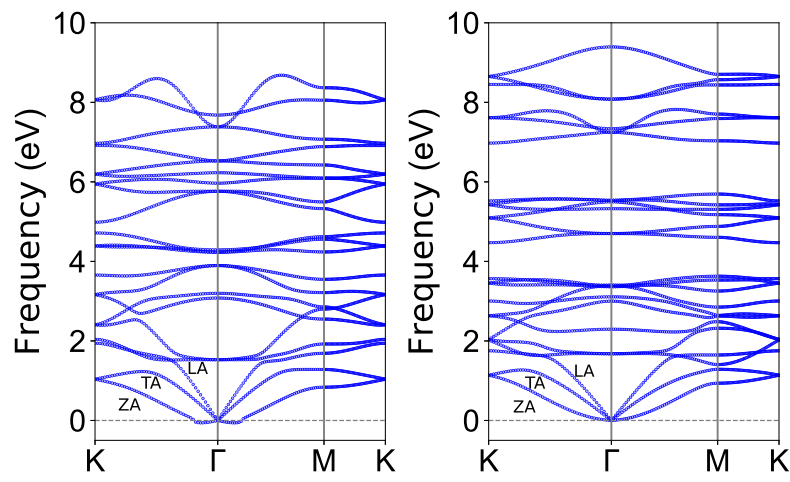

FIG. 13. (Color online)the phonon band dispersions of Janus monolayer $\mathrm{CrF}_{1.5} \mathrm{I}_{1.5}$ (Left) and $\mathrm{CrCl}_{1.5} \mathrm{I}_{1.5}$ (Right) with FM magnetic configuration.

$e_{i j}$ of $\mathrm{CrBr}_{1.5} \mathrm{I}_{1.5}$ monolayer. The calculated $e_{22}$ is $0.119 \times 10^{-10} \mathrm{C} / \mathrm{m}$ with ionic part $0.300 \times 10^{-10} \mathrm{C} / \mathrm{m}$ and electronic part $-0.181 \times 10^{-10} \mathrm{C} / \mathrm{m}$, and $e_{31}$ for $0.432 \times 10^{-10} \mathrm{C} / \mathrm{m}$ with ionic contribution $-0.071 \times 10^{-10}$ $\mathrm{C} / \mathrm{m}$ and electronic contribution $0.503 \times 10^{-10} \mathrm{C} / \mathrm{m}$. It is found that the electronic and ionic polarizations have opposite signs for both $e_{22}$ and $e_{31}$. The ionic contribution to the in-plane piezoelectricity is larger than the electronic contribution. However, the electronic contributions dominate the out-of-plane piezoelectricity. Based on Equation 4, the $d_{22}$ and $d_{31}$ can be attained from previous calculated $C_{i j}$ and $e_{i j}$. The calculated $d_{22}$ and $d_{31}$ are $0.557 \mathrm{pm} / \mathrm{V}$ and $1.138 \mathrm{pm} / \mathrm{V}$. A large outof-plane piezoelectric response is highly desired for $2 \mathrm{D}$ materials, which is compatible with the nowadays bottom/top gate technologies. The $d_{31}$ of $\mathrm{CrBr}_{1.5} \mathrm{I}_{1.5}$ monolayer is obviously higher compared with ones of other 2D known materials, including the oxygen functionalized MXenes (0.40-0.78 pm/V $)^{22}$, Janus TMD monolayers $(0.03 \mathrm{pm} / \mathrm{V})^{5}$, functionalized h-BN $(0.13 \mathrm{pm} / \mathrm{V})^{50}$, kalium decorated graphene $(0.3 \mathrm{pm} / \mathrm{V})^{51}$, Janus groupIII materials $(0.46 \mathrm{pm} / \mathrm{V})^{11}$, Janus BiTeI/SbTeI monolayer $(0.37-0.66 \mathrm{pm} / \mathrm{V})^{52}$ and $\alpha-\mathrm{In}_{2} \mathrm{Se}_{3}(0.415 \mathrm{pm} / \mathrm{V})^{53}$. To the best of our knowledge, the $d_{31}$ is less than one among all pure $2 \mathrm{D}$ materials. So, it is very peculiar that the $d_{31}$ of $\mathrm{CrBr}_{1.5} \mathrm{I}_{1.5}$ monolayer is as high as $1.138 \mathrm{pm} / \mathrm{V}$.

\section{STRAIN EFFECTS}

The strain can effectively tune the electronic structures and piezoelectric properties of $2 \mathrm{D}$ materials ${ }^{13-15}$. Here, we use $a / a_{0}$ to simulate the biaxial strain, where $a$ and $a_{0}$ are the strained and unstrained lattice constants, respectively. To determine the ground state of strained $\mathrm{CrF}_{1.5} \mathrm{I}_{1.5}$ monolayer, four different initial magnetic configurations (Figure 2) are considered. The energy differences of AF-Néel, AF-zigzag and AF-stripy with respect to FM state as a function of strain with rectangle supercell are shown in Figure 6. It is found that a magnetic phase transition can be induced by compressive strain with the critical point being about 0.95 , which implies the robustness of the intrinsic ferromagnetism in $\mathrm{CrF}_{1.5} \mathrm{I}_{1.5}$ monolayer. Calculated results show that $\mathrm{CrF}_{1.5} \mathrm{I}_{1.5}$ monolayer prefers $\mathrm{FM}$ ground state with $a / a_{0}$ being greater than about 0.95 in considered strain range, and the AF-Néel become ground state with $a / a_{0}$ being less than about 0.95. Similar phenomenon can also be found in $\mathrm{CrI}_{3}$ monolayer, and the AF-Néel phase becomes the most stable phase at 0.92 strain $^{32}$.

The energy band structures of FM $\mathrm{CrF}_{1.5} \mathrm{I}_{1.5}$ monolayer with strain from 0.94 to 1.06 except 1.00 are plotted in Figure 7, and the majority-spin, minority-spin and total gaps are shown in Figure 8. It is clearly seen that strained $\mathrm{CrF}_{1.5} \mathrm{I}_{1.5}$ monolayer are all indirect gap semiconductors in considered strain range. It is found that the majority-spin and total gaps coincide except 0.94 strain, which means that $\mathrm{CrF}_{1.5} \mathrm{I}_{1.5}$ monolayer holds halfsemiconductor character. At 0.94 strain, the VBM is at minority-spin channel from previous majority-spin one. From 1.06 to 0.94 strain, strain makes both conduction and valence bands of minority-spin channel move toward Fermi level, which leads to the reduced minority-spin gap. The majority-spin gap shows a nonmonotonic behavior, which is mainly due to change of CBM. In fact, at 0.94 strain, the AF-Néel becomes ground state, and we plot the energy bands along with FM states in Figure 9 using orthorhombic supercell. The AF-Néel state still is an indirect gap semiconductor with the gap value of $1.282 \mathrm{eV}$, and the local magnetic moments of $\mathrm{Cr}$ is $2.893 \mu_{B}$.

The strain engineering has been proved to be an very effective way to enhance piezoelectric properties of $2 \mathrm{D}$ materials, and then the strain effects on piezoelectric properties of $\mathrm{CrBr}_{1.5} \mathrm{I}_{1.5}$ monolayer are performed. The elastic constants including $C_{11}, C_{12}, C_{11}-C_{12}$ and 


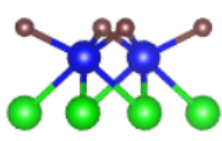

(a)

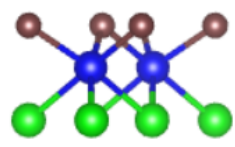

(b)

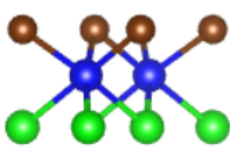

(c)
FIG. 14. (Color online)The side views of crystal structure of $\mathrm{CrF}_{1.5} \mathrm{I}_{1.5}$ (a), $\mathrm{CrCl}_{1.5} \mathrm{I}_{1.5}$ (b) and $\mathrm{CrBr}_{1.5} \mathrm{I}_{1.5}$ (c).

$C_{11}+C_{12}$ of $\mathrm{CrBr}_{1.5} \mathrm{I}_{1.5}$ monolayer with $\mathrm{FM}$ state as a function of biaxial strain are plotted in Figure 10, along with ones of AF-Néel state at 0.94 strain. It is clearly seen that $C_{11}, C_{12}, C_{11}-C_{12}$ and $C_{11}+C_{12}$ are all decreases with increasing strain from 0.94 to 1.06 strain. It is found that $C_{11}, C_{12}, C_{11}-C_{12}$ and $C_{11}+C_{12}$ with AF-Néel state are lower than ones with FM state at 0.94 strain. So, it is important to consider the magnetic configurations for calculating elastic constants. Calculated results show that the $\mathrm{CrBr}_{1.5} \mathrm{I}_{1.5}$ monolayer is mechanically stable in the considered strain range, since the calculated elastic constants satisfy the mechanical stability criteria $^{49}$.

The piezoelectric stress coefficients $\left(e_{22}\right.$ and $\left.e_{31}\right)$ along the ionic and electronic contributions and piezoelectric strain coefficients $\left(d_{22}\right.$ and $\left.d_{31}\right)$ of $\mathrm{CrBr}_{1.5} \mathrm{I}_{1.5}$ monolayer with FM state as a function of biaxial strain are plotted in Figure 11 and Figure 12, along with ones of AF-Néel state at 0.94 strain. It is found that the compressive strain can enhance the $d_{22}$ due to improved $e_{22}$ based on Equation 4, and the $d_{22}$ improves to $0.993 \mathrm{pm} / \mathrm{V}$ at 0.94 strain from unstrained $0.557 \mathrm{pm} / \mathrm{V}$. The tensile strain can decrease the $d_{22}$, and the $d_{22}$ at 1.06 strain reduces to $0.039 \mathrm{pm} / \mathrm{V}$ due to very small $e_{22}\left(0.0066 \times 10^{-10} \mathrm{C} / \mathrm{m}\right)$. For $d_{31}$, the opposite strain dependence is observed, and the tensile strain can improve $d_{31}$ due to reduced $C_{11}+C_{12}$. At 1.06 strain, the $d_{31}$ of $\mathrm{CrBr}_{1.5} \mathrm{I}_{1.5}$ monolayer is $1.545 \mathrm{pm} / \mathrm{V}$, increased by $36 \%$ with respect to unstrained one. In considered strain range, the electronic and ionic parts have opposite signs for both $e_{22}$ and $e_{31}$, and they (absolute value) all decreases with strain from 0.94 to 1.06 .

The magnetic configuration may have important effects on piezoelectric coefficients, and a magnetic phase transition may induce the jump of piezoelectric coefficients. We recalculate the $e_{22}$ and $e_{31}$ along the ionic and electronic contributions and $d_{22}$ and $d_{31}$ of $\mathrm{CrBr}_{1.5} \mathrm{I}_{1.5}$ monolayer with AF-Néel state at 0.94 strain. It is found that magnetic configuration has important effect on $e_{22}$ from $0.285 \times 10^{-10} \mathrm{C} / \mathrm{m}$ of $\mathrm{FM}$ state to $0.181 \times 10^{-10}$ $\mathrm{C} / \mathrm{m}$ of $\mathrm{AF}-\mathrm{Néel}$ state, and has little influence on $e_{31}$ $\left(0.502 \times 10^{-10} \mathrm{C} / \mathrm{m}\right.$ for $\mathrm{FM}$ and $0.495 \times 10^{-10} \mathrm{C} / \mathrm{m}$ for AF-Néel). The similar effects on $d_{22}$ and $d_{31}$ also can be found, and the $d_{22}\left(d_{31}\right)$ changes from $0.993 \mathrm{pm} / \mathrm{V}$ $(0.933 \mathrm{pm} / \mathrm{V})$ of FM state to $0.677 \mathrm{pm} / \mathrm{V}(0.999 \mathrm{pm} / \mathrm{V})$ of AF-Néel state. It is also found that magnetic configuration has important effects on both the electronic and ionic parts of $e_{22}$, and has neglectful influences on ones of $e_{31}$. So, it is very important to consider mag-
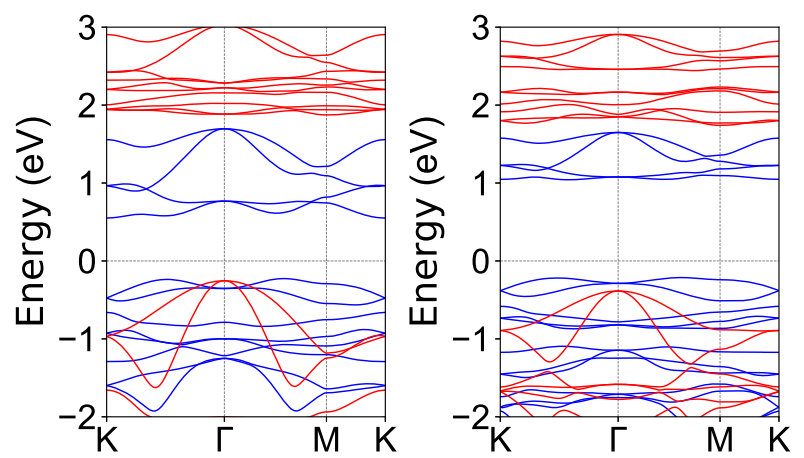

FIG. 15. (Color online)The energy band structures of $\mathrm{CrF}_{1.5} \mathrm{I}_{1.5}$ (Left) and $\mathrm{CrCl}_{1.5} \mathrm{I}_{1.5}$ (Right) with FM state. The blue (red) lines represent the band structure in the spin-up (spin-down) direction.

netic order for piezoelectric coefficients. It is interesting that the PAFM can be induced by compressive strain, which may open up potential opportunities for intriguing physics and novel devices.

\section{DISCUSSION AND CONCLUSION}

In fact, one of two I layers of monolayer $\mathrm{CrI}_{3}$ can also be replaced by $\mathrm{F}$ or $\mathrm{Cl}$ atoms, namely monolayer $\mathrm{CrF}_{1.5} \mathrm{I}_{1.5}$ and $\mathrm{CrCl}_{1.5} \mathrm{I}_{1.5}$. For $\mathrm{CrCl}_{1.5} \mathrm{I}_{1.5}$ monolayer, the FM order still is the ground state by comparing energy difference of four different initial magnetic configurations (Figure 2). However, for monolayer $\mathrm{CrF}_{1.5} \mathrm{I}_{1.5}$, the FM and AF-Néel orders have almost the same energy, and the difference is only $0.48 \mathrm{meV} / \mathrm{CrF}_{1.5} \mathrm{I}_{1.5}$ formula. So, we focus on the FM state of both monolayer $\mathrm{CrF}_{1.5} \mathrm{I}_{1.5}$ and $\mathrm{CrCl}_{1.5} \mathrm{I}_{1.5}$ for a better comparison. For monolayer $\mathrm{CrF}_{1.5} \mathrm{I}_{1.5}\left(\mathrm{CrCl}_{1.5} \mathrm{I}_{1.5}\right)$, the optimized lattice constants is $6.250(6.590) \AA$, and the calculated $C_{11}$ and $C_{12}$ are 49.97 (34.88) $\mathrm{Nm}^{-1}$ and 16.34 (9.94) $\mathrm{Nm}^{-1}$, which satisfy the Born criteria of mechanical stability ${ }^{49}$. From Figure 13, it is proved that monolayer $\mathrm{CrF}_{1.5} \mathrm{I}_{1.5}$ $\left(\mathrm{CrCl}_{1.5} \mathrm{I}_{1.5}\right)$ is dynamically stable. The side views of crystal structures of $\mathrm{CrX}_{1.5} \mathrm{I}_{1.5}(\mathrm{X}=\mathrm{F}, \mathrm{Cl}$ and $\mathrm{Br})$ are plotted in Figure 14, and it is clearly seen that the distortions of octahedral environment located by $\mathrm{Cr}$ atoms become more and more severe with $\mathrm{X}$ from $\mathrm{Br}$ to $\mathrm{Cl}$ to $\mathrm{F}$ due to the more difference in atomic sizes and electronegativities of $\mathrm{X}$ and I atoms. It is found that an easy axis of monolayer $\mathrm{CrCl}_{1.5} \mathrm{I}_{1.5}$ is along the c-direction, and the corresponding MAE is $110 \mu \mathrm{eV}$ per $\mathrm{Cr}$ atom. However, for monolayer $\mathrm{CrF}_{1.5} \mathrm{I}_{1.5}$, an easy axis is along the inplane direction, and the MAE is up to $2151 \mu \mathrm{eV}$ per $\mathrm{Cr}$ atom. Finally, the piezoelectric properties of monolayer $\mathrm{CrF}_{1.5} \mathrm{I}_{1.5}$ and $\mathrm{CrCl}_{1.5} \mathrm{I}_{1.5}$ are investigated, and their $d_{31}$ is up to $2.578 \mathrm{pm} / \mathrm{V}$ and $1.804 \mathrm{pm} / \mathrm{V}$, respectively. The related data are summarized in Table I. In fact, many PFMs can be achieved in $2 \mathrm{D} \mathrm{CrX}_{3}(\mathrm{X}=\mathrm{F}, \mathrm{Cl}, \mathrm{Br}$ and I) family by using the same design principle of mono- 
layer $\mathrm{CrBr}_{1.5} \mathrm{I}_{1.5}$, for example Janus monolayer monolayer $\mathrm{CrCl}_{1.5} \mathrm{~F}_{1.5}, \mathrm{CrCl}_{1.5} \mathrm{Br}_{1.5}, \mathrm{CrBr}_{1.5} \mathrm{~F}_{1.5}$ and so on.

In summary, our theoretical calculations demonstrate that the PFM can occur in Janus $\mathrm{CrBr}_{1.5} \mathrm{I}_{1.5}$ monolayer with dynamic, mechanical and thermal stabilities, which possesses a sizable MAE. By breaking the inversion and mirror symmetry, both in-plane and out-ofplane piezoelectric polarizations can be induced by a uniaxial in-plane strain. Amazingly, the out-of-plane $d_{31}$ $(1.138 \mathrm{pm} / \mathrm{V})$ is obviously higher compared with ones of many familiar 2D materials. It is proved that strain engineering can effectively tune piezoelectricity of monolayer $\mathrm{CrBr}_{1.5} \mathrm{I}_{1.5}$. The PAFM can also be realized by compressive strain, and $d_{22}\left(d_{31}\right)$ is $0.677 \mathrm{pm} / \mathrm{V}(0.999$ $\mathrm{pm} / \mathrm{V})$ at 0.94 strain. Finally, similar to $\mathrm{CrBr}_{1.5} \mathrm{I}_{1.5}$, the PFM can also be achieved in the monolayer $\mathrm{CrF}_{1.5} \mathrm{I}_{1.5}$ and $\mathrm{CrCl}_{1.5} \mathrm{I}_{1.5}$ with very large $d_{31}$ being $2.578 \mathrm{pm} / \mathrm{V}$ and $1.804 \mathrm{pm} / \mathrm{V}$. Our works supply an experimental proposal to achieve large out-of-plane piezoelectric response in PFMs, and hope that the work can stimulate further experimental effort on 2D PFM.

\section{ACKNOWLEDGMENTS}

This work is supported by Natural Science Basis Research Plan in Shaanxi Province of China (2021JM456). We are grateful to the Advanced Analysis and Computation Center of China University of Mining and Technology (CUMT) for the award of CPU hours and WIEN2k/VASP software to accomplish this work.
${ }^{1}$ W. Wu, L. Wang, Y. Li, F. Zhang, L. Lin, S. Niu, D. Chenet, X. Zhang, Y. Hao, T. F. Heinz, J. Hone and Z. L. Wang, Nature 514, 470 (2014).

2 H. Zhu, Y. Wang, J. Xiao, M. Liu, S. Xiong, Z. J. Wong, Z. Ye, Y. Ye, X. Yin and X. Zhang, Nat. Nanotechnol. 10, 151 (2015).

3 A. Y. Lu, H. Zhu, J. Xiao, C. P. Chuu, Y. Han, M. H. Chiu, C. C. Cheng, C. W. Yang, K. H. Wei, Y. Yang, Y. Wang, D. Sokaras, D. Nordlund, P. Yang, D. A. Muller, M. Y. Chou, X. Zhang and L. J. Li, Nat. Nanotechnol. 12, 744 (2017).

${ }^{4}$ M. Dai, Z. Wang, F. Wang, Y. Qiu, J. Zhang, C. Y. Xu, T. Zhai, W. Cao, Y. Fu, D. Jia, Y. Zhou, and P. A. Hu, Nano Lett. 19, 5416 (2019).

${ }^{5}$ L. Dong, J. Lou and V. B. Shenoy, ACS Nano, 11, 8242 (2017).

${ }^{6}$ R. X. Fei, We. B. Li, J. Li and L. Yang, Appl. Phys. Lett. 107, 173104 (2015).

7 M. N. Blonsky, H. L. Zhuang, A. K. Singh and R. G. Hennig, ACS Nano, 9, 9885 (2015).

${ }^{8}$ Y. Chen, J. Y. Liu, J. B. Yu, Y. G. Guo and Q. Sun, Phys. Chem. Chem. Phys. 21, 1207 (2019).

9 S. D. Guo, Y. T. Zhu, W. Q. Mu and W. C. Ren, EPL 132, 57002 (2020).

10 S. D. Guo, Y. T. Zhu, W. Q. Mu, L. Wang and X. Q. Chen, Comp. Mater. Sci. 188, 110223 (2021)

11 Y. Guo, S. Zhou, Y. Z. Bai, and J. J. Zhao, Appl. Phys. Lett. 110, 163102 (2017).

12 W. B. Li and J. Li, Nano Res. 8, 3796 (2015).

13 Dimple, N. Jena, A. Rawat, R. Ahammed, M. K. Mohanta and A. D. Sarkar, J. Mater. Chem. A 6, 24885 (2018).

14 S. D. Guo, X. S. Guo, Y. Y. Zhang and K. Luo, J. Alloy. Compd. 822, 153577 (2020).

15 N. Jena, Dimple, S. D. Behere and A. D. Sarkar, J. Phys. Chem. C 121, 9181 (2017).

16 J. H. Yang, A. P. Wang, S. Z. Zhang, J. Liu, Z. C. Zhong and L. Chen, Phys. Chem. Chem. Phys., 21, 132 (2019).

17 S. D. Guo, W. Q. Mu, Y. T. Zhu and X. Q. Chen, Phys. Chem. Chem. Phys. 22, 28359 (2020).

18 S. D. Guo, W. Q. Mu, Y. T. Zhu, S. Q. Wang and G. Z. Wang, J. Mater. Chem. C, 2021, DOI: 10.1039/D1TC00414J.
19 S. D. Guo, Y. T. Zhu, W. Q. Mu and X. Q. Chen, arXiv:2103.03456 (2021).

20 M. T. Ong and E.J. Reed, ACS Nano 6, 1387 (2012).

21 A. A. M. Noor, H. J. Kim and Y. H. Shin, Phys. Chem. Chem. Phys. 16, 6575 (2014).

22 J. Tan, Y. H. Wang, Z. T. Wang, X. J. He, Y. L. Liu, B. Wanga, M. I. Katsnelson and S. J. Yuan, Nano Energy 65, 104058 (2019).

${ }^{23}$ L. Dong, H. Kumar, B. Anasori, Y. Gogotsi and V. B. Shenoy, J. Phys. Chem. Lett. 8, 422 (2017).

24 Y. Ma, Y. Dai, M. Guo, C. Niu, Y. Zhu and B. Huang, ACS Nano, 6, 1695 (2012).

25 C. Gong, L. Li, Z. Li, H. Ji, A. Stern, Y. Xia, T. Cao, W. Bao, C. Wang, Y. Wang, Z. Q. Qiu, R. J. Cava, S. G. Louie, J. Xia and X. Zhang, Nature 546, 265 (2017).

26 M. Khazaei, M. Arai, T. Sasaki, C. Y. Chung, N. S. Venkataramanan, M. Estili, Y. Sakka and Y. Kawazoe, Adv. Funct. Mater. 23, 2185 (2013).

27 I. Choudhuri, S. Kumar, A. Mahata, K. S. Rawat and B. Pathak, Nanoscale 8, 14117 (2016).

${ }^{28}$ Y. Guo, H. Deng, X. Sun, X. Li, J. Zhao, J. Wu, W. Chu, S. Zhang, H. Pan, X. Zheng, X. Wu, C. Jin, C. Wu and Y. Xie, Adv. Mater. 29, 1700715 (2017).

29 X. M. Wu, Y. L. Feng, S. Li, B. Q. Zhang and G. Y. Gao, J. Phys. Chem. C 124, 16127 (2020).

30 B. Huang, G. Clark, E. Navarro-Moratalla, D. R. Klein, R. Cheng, K. L. Seyler, D. Zhong, E. Schmidgall, M. A. McGuire, D. H. Cobden, W. Yao, D. Xiao, P. JarilloHerrero and X. Xu, Nature 546, 270 (2017).

31 G. Bhattacharyya, I. Choudhuri, P. Bhauriyal, P. Garg and B. Pathak, Nanoscale 10, 22280 (2018).

32 W. B. Zhang, Q. Qu, P. Zhu and C. H. Lam, : J. Mater. Chem. C 3, 12457 (2015).

33 S. Jiang, J. Shan and K. F. Mak, Nat. Mater. 17, 406 (2018).

34 S. Jiang, L. Li, Z. Wang, K. F. Mak and J. Shan, Nat. Nanotechnol. 13, 549 (2018).

35 N. Sivadas, S. Okamoto, X. Xu, C. J. Fennie and D. Xiao, 18, 7658 (2018).

36 Z. Wang, et al., Nat. Commun. 9, 2516 (2018).

37 G. Z. Qin, H. M. Wang, L. C. Zhang, Z. Z. Qin and M. Hu, J. Mater. Chem. C 8, 3520 (2020). 
38 B. Huang, et al., Nat. Nanotechnol. 13, 544 (2018).

39 A. Y. Lu, H. Y. Zhu, J. Xiao et al., Nature Nanotechnology 12, 744 (2017).

40 P. Hohenberg and W. Kohn, Phys. Rev. 136, B864 (1964); W. Kohn and L. J. Sham, Phys. Rev. 140, A1133 (1965).

${ }^{41}$ G. Kresse, J. Non-Cryst. Solids 193, 222 (1995).

42 G. Kresse and J. Furthmüller, Comput. Mater. Sci. 6, 15 (1996).

43 G. Kresse and D. Joubert, Phys. Rev. B 59, 1758 (1999).

44 J. P. Perdew, K. Burke and M. Ernzerhof, Phys. Rev. Lett. 77, 3865 (1996).

45 X. Wu, D. Vanderbilt and D. R. Hamann, Phys. Rev. B 72, 035105 (2005).

46 A. Togo, F. Oba, and I. Tanaka, Phys. Rev. B 78, 134106
(2008).

47 E. Mariani and F. V. Oppen, Phys. Rev. Lett. 100, 076801 (2008).

48 J. Carrete, W. Li, L. Lindsay, D. A. Broido, L. J. Gallego and N. Mingo, Mater. Res. Lett. 4, 204 (2016).

49 R. C. Andrew, R. E. Mapasha, A. M. Ukpong and N. Chetty, Phys. Rev. B 85, 125428 (2012).

50 A. A. M. Noor, H. J. Kim and Y. H. Shin, Phys. Chem. Chem. Phys. 16, 6575 (2014).

51 M. T. Ong and E. J. Reed, ACS Nano 6, 1387 (2012).

52 S. D. Guo, X. S. Guo, Z. Y. Liu and Y. N. Quan, J. Appl. Phys. 127, 064302 (2020).

53 L. Hu and X.R. Huang, RSC Adv. 7, 55034 (2017). 